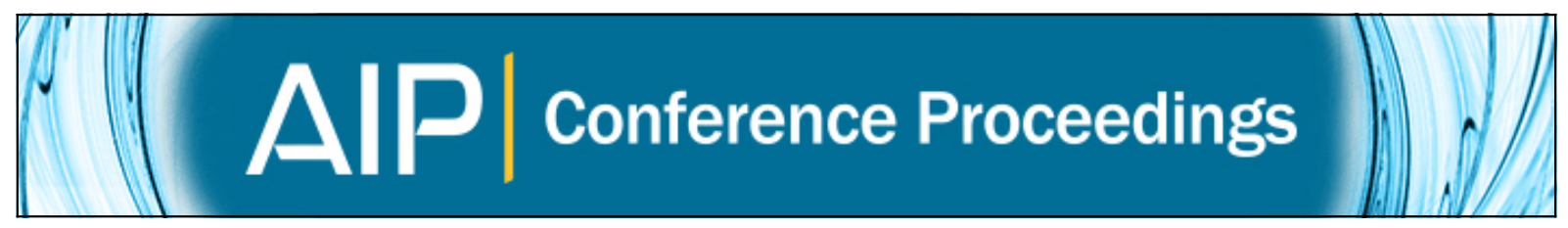

\title{
Guided wave tomography performance analysis
}

Peter Huthwaite, Michael Lowe, and Peter Cawley

Citation: AIP Conference Proceedings 1706, 020021 (2016); doi: 10.1063/1.4940467

View online: http://dx.doi.org/10.1063/1.4940467

View Table of Contents: http://scitation.aip.org/content/aip/proceeding/aipcp/1706?ver=pdfcov

Published by the AIP Publishing

Articles you may be interested in

High resolution guided wave tomography

AIP Conf. Proc. 1511, 729 (2013); 10.1063/1.4789118

GUIDED WAVE TRAVEL TIME TOMOGRAPHY FOR BENDS

AIP Conf. Proc. 1335, 223 (2011); 10.1063/1.3591860

THE APPLICATION OF GUIDED WAVE TRAVEL TIME TOMOGRAPHY TO BENDS

AIP Conf. Proc. 1211, 774 (2010); 10.1063/1.3362474

EXPERIMENTAL RESULTS OF GUIDED WAVE TRAVEL TIME TOMOGRAPHY

AIP Conf. Proc. 1211, 2052 (2010); 10.1063/1.3362375

Guided wave helical ultrasonic tomography of pipes

J. Acoust. Soc. Am. 114, 767 (2003); 10.1121/1.1593068 


\title{
Guided Wave Tomography Performance Analysis
}

\author{
Peter Huthwaite ${ }^{1, \text { a) }}$, Michael Lowe ${ }^{1}$ and Peter Cawley ${ }^{1}$ \\ ${ }^{1}$ Department of Mechanical Engineering, Imperial College London, SW7 2AZ, London, UK

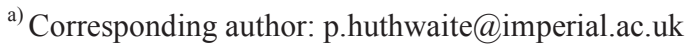

\begin{abstract}
Quantifying wall loss caused by corrosion is a significant challenge for the petrochemical industry. Corrosion commonly occurs at pipe supports, where surface access for inspection is limited. Guided wave tomography is pursued as a solution to this: guided waves are transmitted through the region of interest from an array, and tomographic reconstruction techniques are applied to the measured signals in order to produce a map of thickness. There are many parameters in the system which can affect the performance; this paper investigates how the accuracy varies as defect width and depth, operating frequency and guided wave mode are all changed. For the S0 mode, the best performance was seen around $170 \mathrm{kHz}$ on the $10 \mathrm{~mm}$ plate, with poor performance seen at almost all other frequencies. A0 showed better performance across a broad range of frequencies, with resolution improving with frequency as the wavelength reduced. However, it was shown that the resolution limit did drop relative to the wavelength, limiting the performance at high frequencies slightly.
\end{abstract}

\section{INTRODUCTION}

Determining the remaining wall thickness of pipes and pressure vessels in the presence of corrosion is of major importance to the petrochemical industry, in order to avoid unexpected ruptures and leaks. In many cases manual ultrasonic thickness gauging techniques are unsuitable because access to the surface is restricted, for example at supports. This is particularly challenging because corrosion is most likely to occur where water can collect near the pipe surface, which will often happen at inaccessible locations such as supports.

Guided wave tomography provides a potential solution to enable thickness maps to be produced in inaccessible areas. Guided Lamb waves are transmitted through the region of interest from a transmitter array, and measured by a receiver array; by some form of inversion process the thickness of the plate can then be determined from the measurements. One of the most widely used approaches is to exploit the dispersive nature of the guided waves, where velocity is a function of frequency; the velocity is reconstructed, and this is then converted to thickness using the known dispersion function. This approach has been investigated by several researchers [1]-[3], although other techniques have been proposed such as the use of Mindlin theory [4] and shear-horizontal modes, which have cutoffs at particular frequency-thickness products [5].

In guided wave tomography, there are a wide range of parameters which can affect the performance of the inversion approach, including the operating frequency and the mode shape, which can generally both be controlled by the system; it is noted that the effect of the different modes and frequencies on the propagation of the guided waves when there were different surface loadings has been investigated [6], but no assessment was undertaken on the underlying imaging performance. This performance will also be affected by the shape of the defect being imaged, which should also be assessed.

This paper aims to evaluate the performance across these different parameters. Initially, the testing method will be outlined, then the results presented and analysed to confirm the trends. Finally conclusions will be drawn.

\section{THEORY}

Using the standard guided wave tomography approach, the initial step is to produce a velocity map from the measurements. For these purposes HARBUT (Hybrid Algorithm for Robust Breast Ultrasound Tomography) [7] was exploited. This was initially developed for breast imaging, and combines the complementary strengths of bentray tomography and diffraction tomography to determine low- and high-spatial frequency components of the image respectively. Based on this, the technique is shown to reconstruct velocity maps down to a resolution of around halfa-wavelength [8], matching the standard Born approximation limitation. However, the resolution when 
reconstructing thickness maps was significantly lower than this, which is a result of inaccuracies in the underlying assumption that guided waves scatter from thickness variations in the same way that acoustic waves scatter from velocity variations [8]. It is this assumption which is primarily investigated through this paper. Because the performance is not limited by HARBUT, but rather this underlying assumption, the results will generalise to other velocity reconstruction techniques, provided they have sufficiently good performance. This is because they will be unable to bypass the fundamental limit imposed by the thickness-velocity mapping.

\section{METHOD}

For the purposes of this study a simple axisymmetric Hann-shaped function is used. Although more complex defects are likely to result from surface corrosion, it should be noted from other work [8] that the behaviour for these complex shapes is likely to be very similar to a superposition of simpler defects. The plate thickness, $t(\mathbf{x})$ in the presence of this defect is described mathematically by

$$
t(\mathbf{x})=\left\{\begin{aligned}
t_{0}-\frac{d}{2}\left[1+\cos \left(\frac{2 \pi}{w}|\mathbf{x}|\right)\right], & |\mathbf{x}| \leq \frac{w}{2} \\
t_{0}, & |\mathbf{x}|>\frac{w}{2}
\end{aligned}\right.
$$

where $t_{0}$ is the background thickness, $d$ is the defect depth, $w$ is the defect width. For the purposes of this study, the nominal plate thickness $t_{0}$ was taken as $10 \mathrm{~mm}$, although the results will generalise to other thicknesses by selecting alternative frequencies.

It is then important to produce test data. One option would be to conduct a series of experimental measurements, but this would be quite impractical given the huge number of defect widths and depths which are of interest. Instead, a simulation approach is proposed. In this, it is important that the full physics of the guided wave scattering from varying thickness is modelled appropriately. The finite element method is a good choice to do this, and has been validated [8]. Here, the Pogo software package (available from www.pogo-fea.com, [9]) was used; this is a high speed explicit time-domain solver running on graphics cards.

The time-domain simulation enables many frequencies to be simulated simultaneously. Therefore, a very broadband chirp excitation was used to excite the signal, and a deconvolution approach used in post-processing to enable different toneburst signals to be extracted from the resulting measurements. This signal is described as

$$
\mathrm{s}(\mathrm{t})=\sin \left\{2 \pi \mathrm{t}\left[f_{\text {low }}+\frac{\left(\mathrm{t}-t_{0}\right)\left(f_{\text {high }}-f_{\text {low }}\right)}{\mathrm{t}_{\text {sig }}}\right]\right\}
$$

running from $t_{0}$ for time $t_{\text {sig }}$, with $f_{\text {high }}$ and $f_{\text {low }}$ being the extreme frequency values of interest.

In these cases, the defect depths were varied from $10 \%$ to $80 \%$ of wall thickness in $10 \%$ increments, and the defect width was set to $24,16,10,8,7,6,5,4,3,2,1.5$ and 1 times the plate thickness. For the A0 mode (excited by applying an out-of-plane force to a through-thickness column of nodes) frequencies $15-150 \mathrm{kHz}$ were simulated, and for S0 these were $100-250 \mathrm{kHz}$. In the case of the former, since the A0 group velocity dispersion curve is nonmonotonic, determining thickness from velocity reliably was found to be very difficult above $130 \mathrm{kHz}$, so no data is presented for A0 above this limit.

Once the simulations had been performed, the images were produced using HARBUT, as described previously. The error was then determined at the origin, the deepest point of the true defect; this error was the difference between the reconstructed thickness and the true thickness, and was presented as a fraction of the background plate thickness.

\section{RESOLUTION ANALYSIS}

In guided wave tomography, resolution is one of the most important elements to achieving accurate reconstructions, yet the limit is typically of similar order to most defects [8]. Prior to analysing the performance, therefore, a study of how a particular resolution limit is likely to affect the accuracy of the reconstruction is undertaken. Two key assumptions are made, firstly that a resolution limit behaves like a low-pass filter, removing all spatial frequency components in the image above a certain value which corresponds to that resolution limit. 
Secondly, it is assumed that the mapping between thickness and the underlying object function (the function to which the filter is applied) is linear, so that the filter can effectively be applied to the thickness map itself. While the former assumption is likely to hold for many imaging approaches (this holds, for example, in diffraction tomography [10]) the linear mapping assumption is less likely to be valid, given the non-linear nature of the dispersion curve and the typical object functions which are commonly reconstructed.

Based on these assumptions, the effect on accuracy of filtering simple defect shapes is performed. This is done both with the Hann-shaped defects considered in this paper, as well as a square function, as the width varied relative to the resolution limit. The results are plotted in Fig. 1.

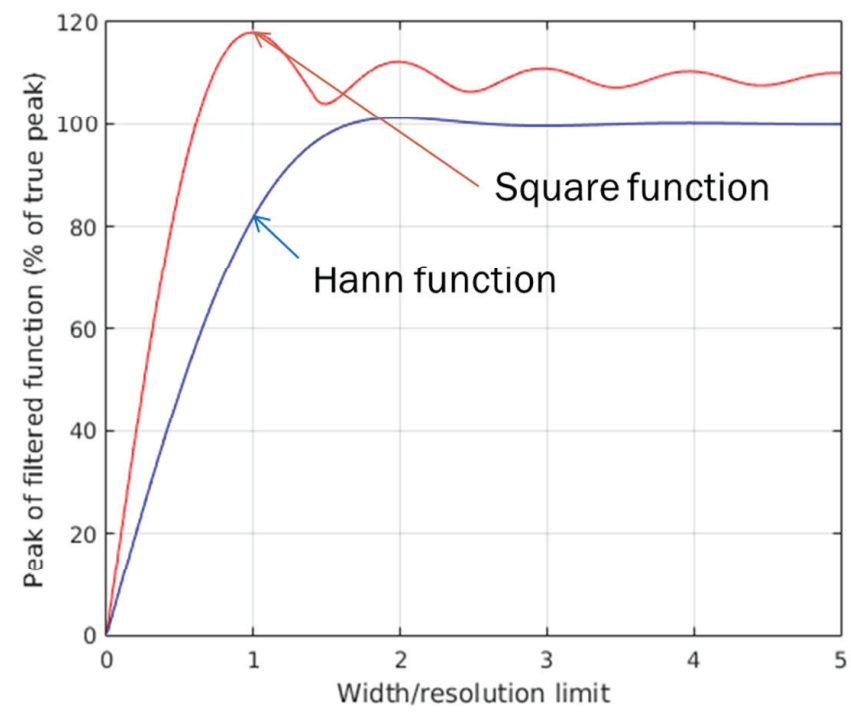

FIGURE 1. Peak depth reconstruction values as a fraction of the true depth as the resolution limit varies.

The Hann function clearly shows a drop-off in accuracy for widths around and below the resolution limit, as the defect wall loss is underestimated. By contrast, a slightly counter-intuitive result is shown for the square function, where the wall loss is overestimated instead. This is caused by the filtered version of the function overshooting that of the true function at the centre.

Focus is now given to the Hann shapes, which are likely to be more representative of real defects. The curve can be approximated, as shown in Fig. 2. 


\section{Corner width,}

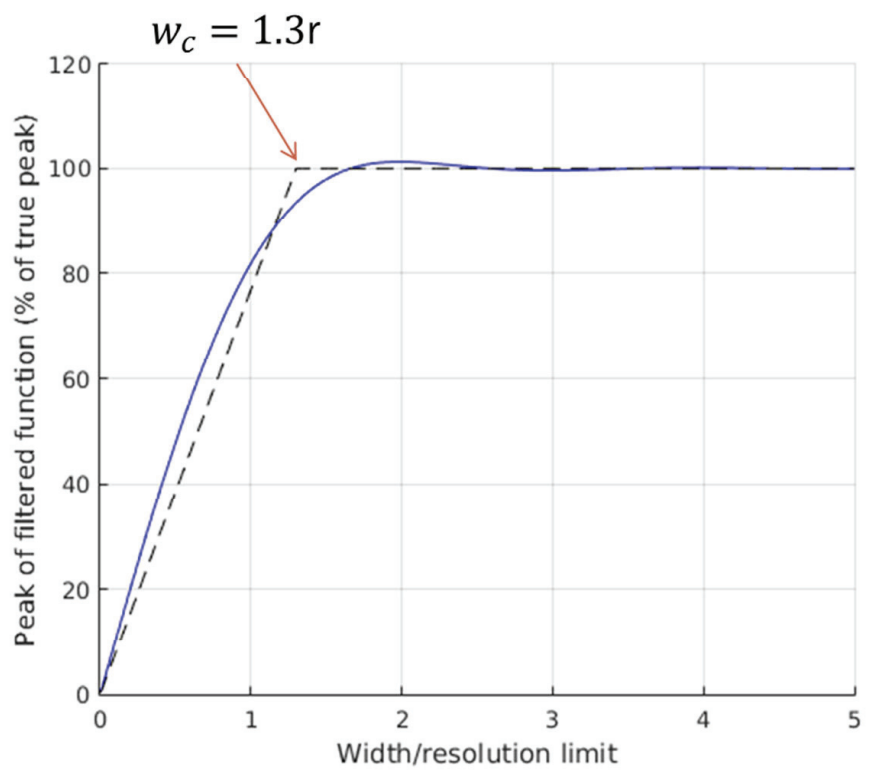

FIGURE 2. Approximating the accuracy loss as the resolution limit changes with a piecewise linear function (dashed black line) compared to the true values (solid blue). The blue curve is the same as in Fig. 1.

Here a corner width, $w_{c}$ is defined as 1.3 times the resolution limit. It can be assumed that defects wider than this will be reconstructed without an error caused by lack of resolution, while defects narrower are expected to have an error estimated as

$$
e \approx d\left(1-\frac{w}{w_{c}}\right)
$$

This provides a simple method for estimating error for a particular defect width, depth and resolution limit.

\section{RESULTS}

\section{A0 Mode}

There are four parameters investigated: width, depth, frequency and mode. Initially, two of these are fixed; the defect width is fixed to $80 \mathrm{~mm}$ ( 8 times the wall thickness) and the A0 mode is taken. The $80 \mathrm{~mm}$ defect was shown previously to be close to the resolution limit so this provides a good opportunity to test the limits of the tomography system. The results are shown in Fig. 3. 

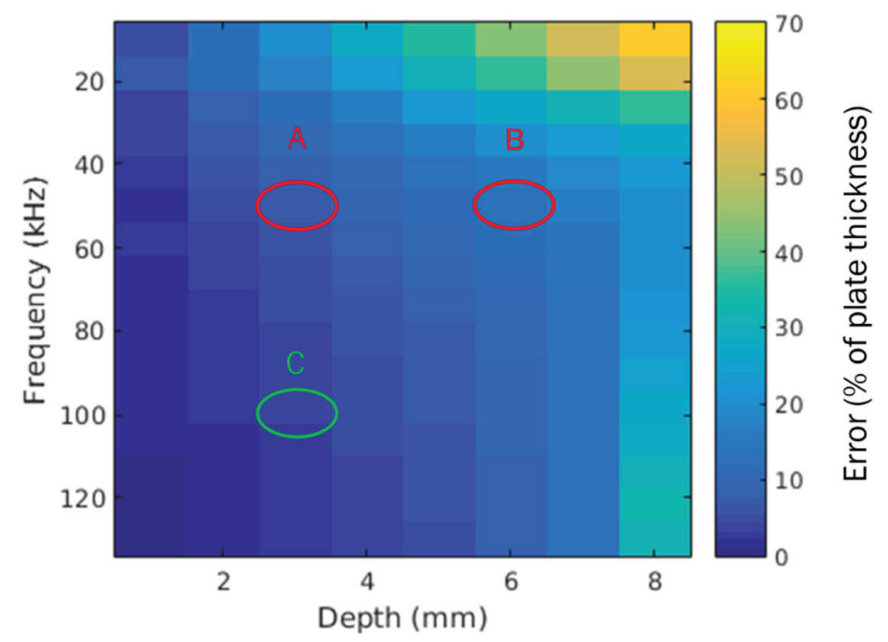

FIGURE 3. Error for the $\mathrm{A} 0$ mode as the frequency and defect depth are varied, for a defect $80 \mathrm{~mm}$ wide on the $10 \mathrm{~mm}$ thick plate.

As a broad picture, the error here seems to increase as defect depth increases, and reduce as frequency increases. These two elements can be investigated by looking at the highlighted points A, B and C. Firstly, comparing at a single frequency, $50 \mathrm{kHz}$, at $3 \mathrm{~mm}$ and $6 \mathrm{~mm}$ deep defects (points A and B) we obtain Fig. 4.
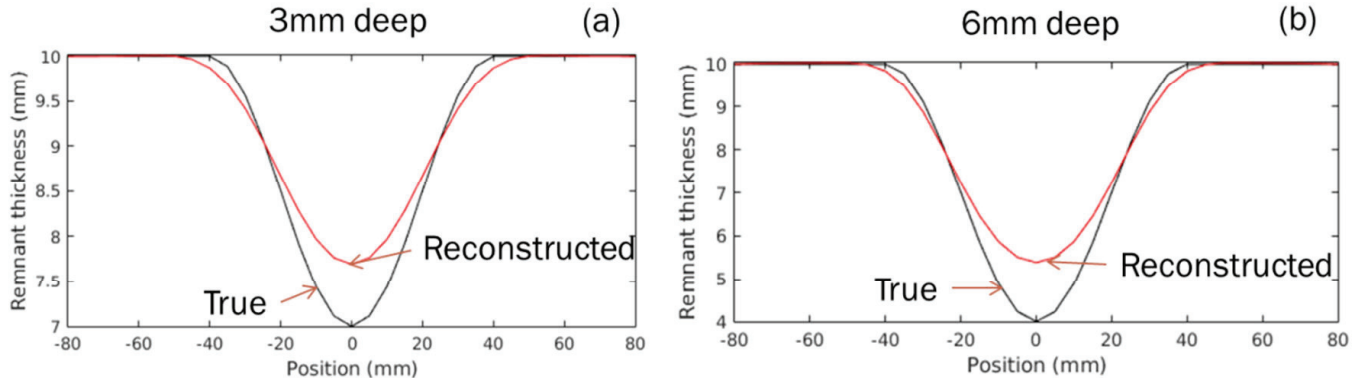

FIGURE 4. Comparing reconstructions using the $\mathrm{A} 0$ mode at $50 \mathrm{kHz}$ with (a) $3 \mathrm{~mm}$ and (b) $6 \mathrm{~mm}$ deep defects, both of which are $80 \mathrm{~mm}$ wide.

It is notable here that the thickness axis is scaled in proportion to the true defect depth, and expressed in this way, both sets of curves appear very similar. In this case, the error is therefore proportional to the depth of the defect, a trend which appears common across all the frequencies plotted in Fig. 3.

To study what happens as frequency varies, cross-sections from the $3 \mathrm{~mm}$ deep defect at $50 \mathrm{kHz}$ are compared to those at $100 \mathrm{kHz}$ in Fig. 5.

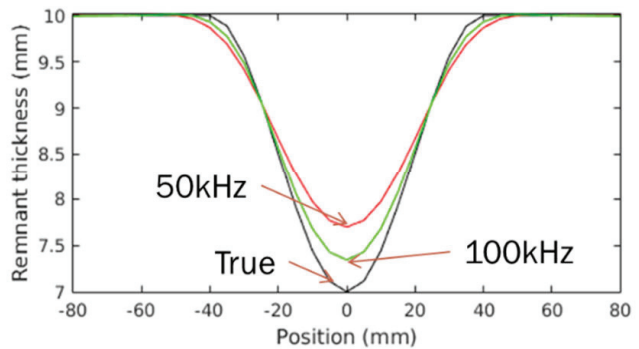

FIGURE 5. Cross sections of the reconstructions for a $3 \mathrm{~mm}$ deep, $80 \mathrm{~mm}$ wide defect, with the A0 mode at $50 \mathrm{kHz}$ and $100 \mathrm{kHz}$. 
This plot shows that the reconstruction's fidelity appears to improve at higher frequencies. This is primarily caused by the improvement in resolution which would be expected at higher frequencies. To analyse this, it is possible to use the formula derived earlier to relate error to the defect depth, width and resolution limit, in order to estimate what the resolution limit is for a particular setup. By taking the case of a $30 \%$ deep defect, whose width is equal to the resolution limit, the error will be approximately $7 \%$, using eq. (1). This means that to find a $30 \%$ defect whose width is equal to the resolution limit, the point where the error is $7 \%$ must be found; it is straightforward to interpolate the data collected across all the different frequencies, widths and depths in order to extract the resolution. This is plotted in Fig. 6(a)

(a)

(b)
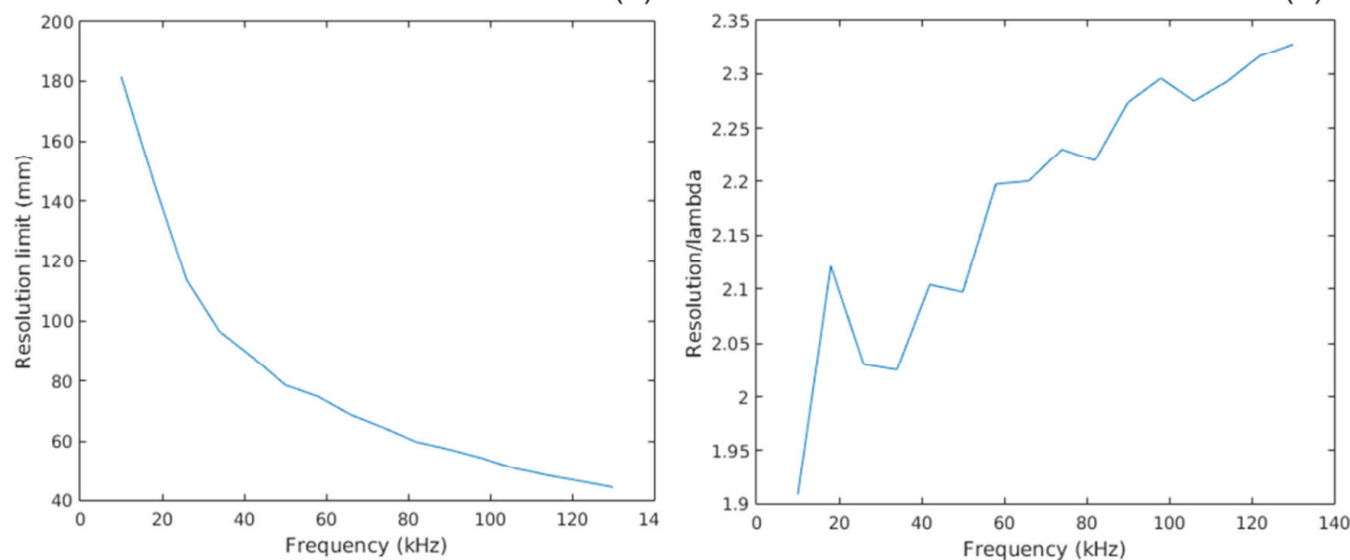

FIGURE 6. Resolution limit for the A0 mode as frequency varies. (a) presents this as an absolute value, and (b) relative to wavelength.

As can be seen, the resolution clearly improves with frequency. This would be expected because wavelength reduces, and the resolution limit for many imaging techniques will often by proportional to the wavelength[10]. By plotting this instead relative to the wavelength, as shown in Fig. 6(b) it can clearly be seen that the resolution is not a constant number of wavelengths and in fact gets worse at higher frequencies. 


\section{S0 Mode}

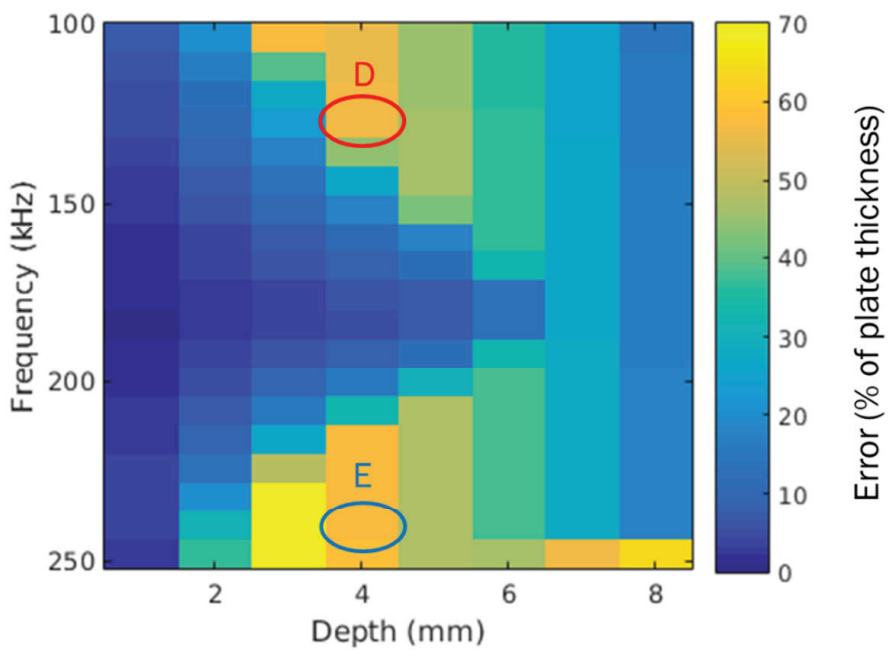

FIGURE 7. Error across different defect depths and frequencies for an $80 \mathrm{~mm}$ wide defect with the S0 mode.

Figure 7 shows the results for an $80 \mathrm{~mm}$ wide defect imaged with the S0 mode. There is a clear region around $170-180 \mathrm{kHz}$ where the accuracy is good, however, away from this there are significant errors in the reconstructions. These errors can be understood by studying the cross section for a $4 \mathrm{~mm}$ deep defect at $130 \mathrm{kHz}$ and $240 \mathrm{kHz}$. Fig. 8 (a) shows these at $130 \mathrm{kHz}$.
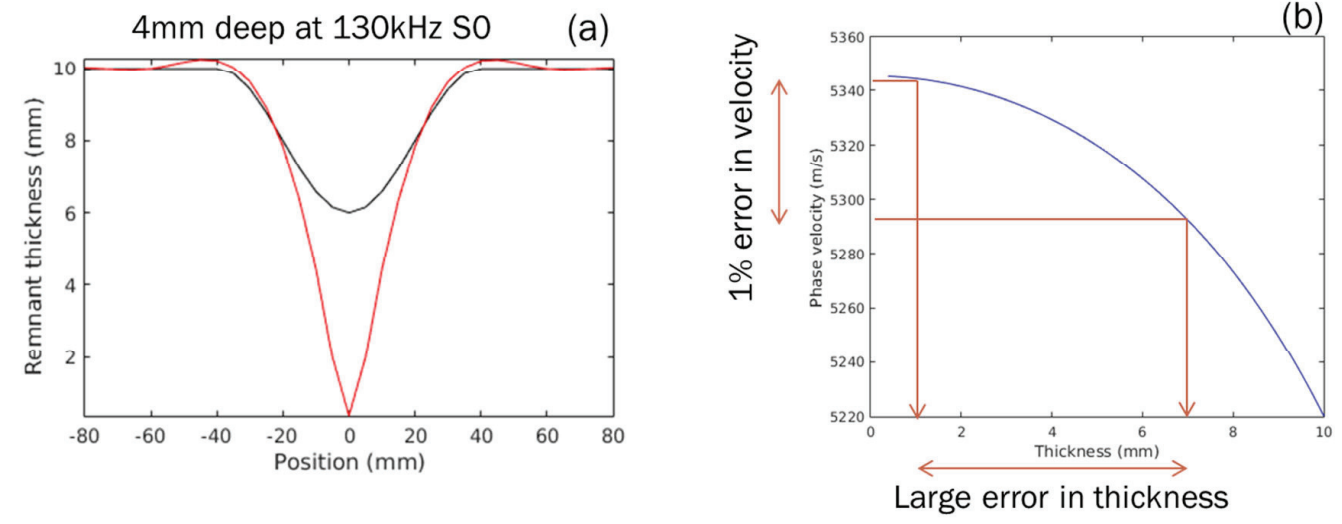

FIGURE 8. (a) shows the cross section of the reconstructed curve (red) and the true curve (black). (b) shows the effect a $1 \%$ velocity error would have on the thickness reconstruction.

The cause of the large thickness error is the very flat dispersion curve at this point, so, as illustrated in Fig. 8(b), a $1 \%$ error in velocity reconstruction can correspond to an error in thickness reconstruction of around $6 \mathrm{~mm}$. However, at higher frequencies, the dispersion curve will be steeper, so it may be expected that the error will not be as severe at these points. While this is true up to $170 \mathrm{kHz}$ from Fig. 7 , beyond this point the error starts to increase again. 

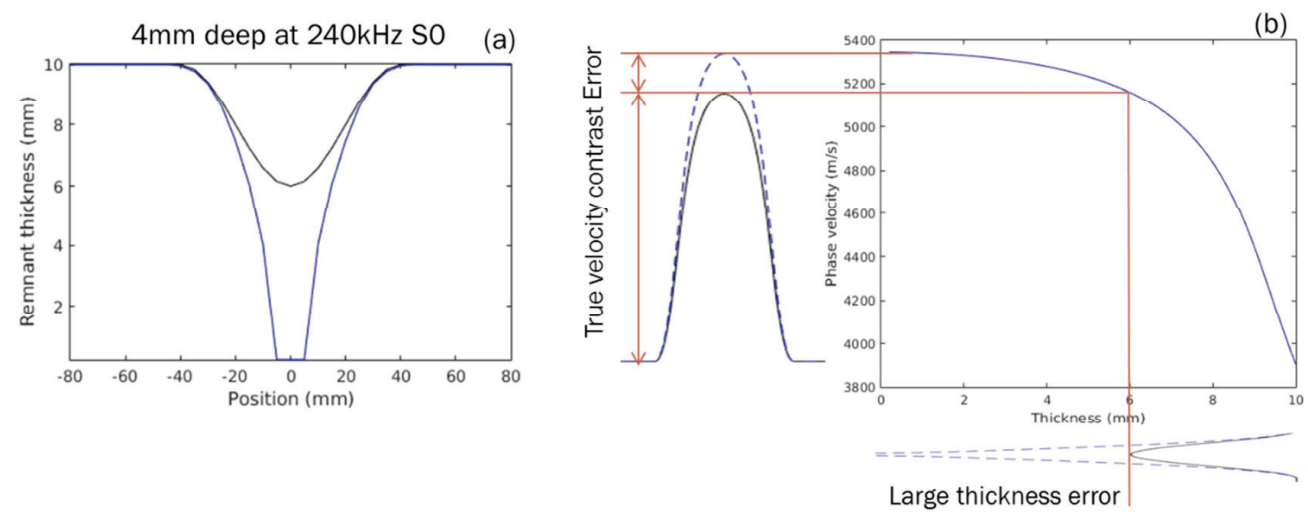

FIGURE 9. (a) shows the cross section of the reconstructed curve (blue) and the true curve (black). (b) shows how a small fractional error on the velocity can saturate the thickness axis due to the highly non-linear dispersion curve.

The result at $240 \mathrm{kHz}$ is plotted in Fig. 9(a), and Fig. 9(b) presents an explanation for this behaviour. Here the error is proportional to the velocity contrast. However, because of the significant non-linearity of the dispersion curve, the error is projected along the flattest region of the curve, and therefore effectively saturates the thickness axis and causes the very large error in the reconstruction.

\section{CONCLUSIONS}

This paper has investigated the inaccuracies of guided wave tomography for a wide range of different defect widths and depths, across the A0 and S0 modes, as the frequency was varied. For A0, the performance was shown to be good throughout the whole bandwidth, with error being proportional to defect depth but reducing as frequency increased. However, the effective resolution improvement was lower than the reduction in wavelength. The S0 mode showed poor performance across most frequencies. At lower frequencies this was caused by the dispersion curve being very shallow, so that a small velocity error would cause a large thickness error. At higher frequencies where the dispersion curve was steeper, the large velocity contrast associated with the steep curve would cause a large proportional error, which would then be projected onto the relatively shallow section of the dispersion curve. Between these points, around $170-180 \mathrm{kHz}$, the best performance was seen, with good reconstructions down to around $50 \%$ wall loss.

\section{ACKNOWLEDGEMENTS}

The authors would like to acknowledge funding from the Engineering and Physical Sciences Research Council, grant numbers EP/H040072/1, EP/H045430/1, EP/M020207/1, and Petrobras and Shell via the UK Research Centre in NDE (RCNDE).

\section{REFERENCES}

1. A. Volker, A. Mast, and J. Bloom, "Experimental results of guided wave travel time tomography," in Review of Progress in Quantitative Nondestructive Evaluation, eds. D. O. Thompson and D. E. Chimenti (American Institute of Physics 1211, Melville, NY) 29, 2052-2059 (2010).

2. P. Belanger and P. Cawley, "Feasibility of low frequency straight-ray guided wave tomography," NDT E Int., 42 (2), 113-119 (2009).

3. P. Huthwaite and F. Simonetti, "High resolution guided wave tomography," Wave Motion, 50 (5), 979-993 (2013).

4. L. R. F. Rose and C. H. Wang, "Mindlin plate theory for damage detection: imaging of flexural inhomogeneities.," J. Acoust. Soc. Am., 127 (2), 754-63 (Feb. 2010). 
5. P. Belanger, "High order shear horizontal modes for minimum remnant thickness.," Ultrasonics, 54 (4), 10781087 (Apr. 2014).

6. P. Huthwaite, R. Ribichini, P. Cawley, and M. J. S. Lowe, "Mode selection for corrosion detection in pipes and vessels via guided wave tomography.," IEEE Trans. Ultrason. Ferroelectr. Freq. Control, 60 (6), 1165-1177 (June 2013).

7. P. Huthwaite and F. Simonetti, "High-resolution imaging without iteration: a fast and robust method for breast ultrasound tomography.," J. Acoust. Soc. Am., 130 (3), 1721 (Sept. 2011).

8. P. Huthwaite, "Evaluation of inversion approaches for guided wave thickness mapping," Proc. R. Soc. A Math. Phys. Eng. Sci., 470 (2166), 20140063-20140063 (Apr. 2014).

9. P. Huthwaite, "Accelerated finite element elastodynamic simulations using the GPU," J. Comput. Phys., 257 (A), 687-707 (2014).

10. A. C. Kak and M. Slaney, Principles of computerized tomographic reconstruction. New York: IEEE Press, 1998. 